Pacific Journal of Mathematics

SQUARE INTEGRABLE PRIMARY REPRESENTATIONS 


\title{
SQUARE INTEGRABLE PRIMARY REPRESENTATIONS
}

\author{
Calvin C. Moore
}

If $\pi$ is a unitary representation of a locally compact group $G$, a weight $\phi$ on the von Neumann algebra $R(\pi)$ generated by $\pi$ is called semi-invariant if it is transformed into scalar multiples of itself by the action of $G$. If $\pi$ is primary we show such objects are essentially unique if one specifies the scaling factor (Schur's lemma). We then study square integrable primary representations and show that a number of possible different definitions are equivalent. We show that any such representation $\pi$ has a semi-invariant weight scaling by the modular function, and this object is seen to be the proper generalization of the formal degree of $\pi$. We formulate and prove generalized Schur orthogonality relations for $\pi$. Finally we specialize to the semi-finite case and identify the formal degree as an operator affiliated to $R(\pi)$. For irreducible $\pi$ the results reduce to those of Duflo and the author, and Phillips.

(1) Let $G$ be a separable (second countable) locally compact group with center $Z$, and let $\pi$ be an irreducible unitary representation of $G$. One then says that $\pi$ is square integrable (in the wide sense) if there are non-zero vectors $x, y$ in the Hilbert space $H_{\pi}$ so that the absolute value of the matrix coefficient $|(x, \pi(g) y)|$ is a square integrable function on the quotient group $G / Z$. That $|(x, \pi(g) y)|$ depends only on the $Z$ coset of $g$ for an irreducible (or more generally, a primary) representation $\pi$ is clear since $\pi(g z)=\lambda_{\pi}(z) \pi(g)$ for some continuous homomorphism $\lambda_{\pi}$ of $Z$ into the circle group $T$. We say that $\pi$ is associated to $\lambda_{\pi}$. The representation $\pi$ is square integrable (in the strict sense) if $(x, \pi(g) y)$ is square integrable on $G$ for some non-zero $x$ and $y$. If $Z$ is compact the two notions coincide, and in general all questions of substance concerning such representations can be reduced to the strict sense case. To see this, note that $\lambda_{\pi}$ is a homomorphism of $Z$ into $T$ and therefore corresponding to the group extension of the group $G / Z$ by $Z$, we can construct a corresponding group extension of $G / Z$ by $T$, say $G^{*}$. There is a bijective correspondence between all primary representations $\pi$ of $G$ associated to $\lambda_{\pi}$ and all primary representations of $G^{*}$ associated to the homomorphism of $T$ into itself, given by the identity map, so that in addition square integrability in the wide sense is preserved. But for $G^{*}$ wide sense square integrability is the same as 
strict sense square integrability. Therefore for simplicity of statement we will talk about strict sense square integrability.

For unimodular groups $G$ and irreducible representations $\pi$, the phenomenon of square integrability has been well understood for many years; the standard results are that if one matrix coefficient is square integrable, all are, and this condition is equivalent to $\pi$ being a summand of the regular representation. A key fact is that the matrix coefficients satisfy orthogonality relations which have the form

$$
\int(x, \pi(g) u)(\overline{y, \pi(g) v}) d g=\frac{1}{d_{\pi}}(x, y)(\overline{u, v})
$$

where $d_{\pi}$ is a positive number called the formal degree. It of course depends on the normalization of Haar measure and if $d g$ is multiplied by $\lambda>0, d_{\pi}$ has to be multiplied by $\lambda^{-1}$. The reader is referred to [5] for a more general formulation of these results in the context of Hilbert algebras. Recently M. Duflo and the author [2] found appropriate generalizations of these results when $G$ is no longer required to be unimodular (cf. also [4]). The key observation was to realize that the formal degree $d_{\pi}$ had to be replaced by a positive self-adjoint operator $D_{\pi}$ which is semi-invariant for the group in that $\pi(g) D_{\pi} \pi(g)^{-1}=\Delta(g) D_{\pi}$ where $\Delta$ is the modular function of $G$. Then square integrability means that some matrix coefficient is square integrable and this is equivalent to being a summand of the regular representation; but it is no longer true that all matrix coefficients are square integrable; more precisely, $(x, \pi(g) u)$ is square integrable with respect to left Haar measure on $G$ iff $u$ is in the domain of $D_{\pi}^{-1 / 2}$ and then we have orthogonality relations of the form

$$
\int(x, \pi(g) u)(\overline{y, \pi(g) v}) d \mu_{l}(g)=(x, y)\left(D_{\pi}^{-1 / 2} u, D_{\pi}^{-1 / 2} v\right)
$$

where $\mu_{l}$ is left Haar measure (Theorem 3 of [2]).

It is our object here to investigate the same kinds of questions for factor representations $\pi$. These questions were inspired by work of Rosenberg [6], and we profited from conversations with him. Of course if $\pi$ is type I, all questions are trivially reduced to the case $\pi$ irreducible, but if $\pi$ is type II, the situation is not entirely transparent, and if $\pi$ is type III, in which case $G$ is forced to be nonunimodular, a new phenomenon occurs which bears further study. In particular there is no way to define a formal degree operator and in fact we shall see that a formal degree operator exists if and only if the von Neumann algebra $R(\pi)$ generated by $\pi$ is semi-finite. Thus our first task is to find the appropriate notion 
of the formal degree of a representation $\pi$ which will reduce to the correct thing in case $R(\pi)$ is semi-finite. We shall find this extension by employing the notion of semi-invariant weights on von Neumann algebras.

(2) Let $R$ be a von Neumann algebra; and $\phi$ a weight on $R$. All weights considered will be normal and semi-finite. Now suppose that $R=R(\pi)$ is the von Neumann algebra generated by a primary representation $\pi$ of a locally compact separable group $G$ and let $\lambda$ be a continuous homomorphism of $G$ into the positive real numbers.

Definition. Let $\phi$ be a nonzero normal semi-finite weight on $R(\pi)$; we say that $\phi$ is semi-invariant of degree $\lambda$ if $\phi\left(\pi(g) x \pi(g)^{-1}\right)=$ $\lambda(g) \phi(x)$ for $x \in R(\pi)^{+}$.

One's intuition suggests that if such an object exists at all for a given $\lambda$, that it should be unique up to scalar multiples since $R(\pi)$ is a factor. This is in fact the case but we first need the following fact.

Proposition 2.1. A semi-invariant nonzero weight $\phi$ is necessarily faithful.

Proof. Let $N_{\phi}=\left\{x: \phi\left(x^{*} x\right)=0\right\}$. Then $N_{\phi}$ is a $\sigma$-weak closed left ideal and if $x \in N_{\phi}, \quad \phi\left((x \pi(g))^{*} x \pi(g)\right)=\lambda(g)^{-1} \phi\left(x^{*} x\right)=0 \quad$ so $x \pi(g) \in N_{\phi}$. Since the $\pi(g)$ generate $R(\pi)$, and $N_{\phi}$ is $\sigma$-weak closed, it is then a two-sided ideal, hence 0 or $R(\pi)$. Since $\phi \neq 0$, it is zero, hence $\phi$ is faithful.

THEOREM 1. If $\phi$ and $\psi$ are two nonzero semi-finite normal weights on $R(\pi)$ semi-invariant of degree $\lambda$, then $\psi=a \phi$ for some $a>0$.

Proof. We employ the Radon-Nikodym derivative $(D \psi: D \phi)_{t}=$ $u_{t}^{\psi, \phi}$ of $\psi$ with respect to $\phi$ as defined in [1]. If $u$ is a unitary operator in $R(\pi)$ it follows from the chain rule satisfied by Radon-Nikodym derivatives that

$$
\left(D \psi^{u}: D \phi^{u}\right)_{t}=u^{*}(D \psi: D \phi)_{t} u
$$

where $\psi^{u}(x)=\psi\left(u x u^{*}\right)$. Moreover, since the modular automorphism group corresponding to $\lambda \phi$ is the same as that corresponding to $\phi$, it follows again from the properties of Radon-Nikodym derivatives that for $\lambda>0,(D \lambda \phi: D \phi)_{t}=\exp (i t(\log \lambda)) 1$. By using the chain rule again we conclude that $(D \lambda \psi: D \lambda \phi)_{t}=(D \psi: D \phi)_{t}$. We now put $u=\pi(g)$ and 
conclude that

$$
\begin{gathered}
\pi(g)^{*}(D \psi: D \phi)_{t} \pi(g)=\left(D \psi^{\pi(g)}: D \phi^{\pi(g)}\right) \\
=(D \lambda(g) \psi: D \lambda(g) \phi)_{t}=(D \psi: D \phi)_{t} .
\end{gathered}
$$

Consequently $(D \psi: D \phi)_{t}$ commutes with $\pi(g)$ and since the operators $\pi(g)$ generate the factor $R(\pi),(D \psi: D \phi)_{t}$ is central and hence a multiple of the identity. By the cocycle property of these functions, it follows now that $(D \psi: D \phi)_{t}$ is a one-parameter unitary group and so $(D \psi: D \phi)_{t}=\exp (+i(\log a) t) 1$ for some $a>0$. But we have already noted that $(D a \phi: D \phi)_{t}=\exp (+i \log (a) t) 1$ and hence by the unicity part of Theorem 1.2.4 of [1] it follows that $\psi=a \phi$.

We shall see that such an object will be the proper one to play the role of the formal degree in the general setting. A more restricted (as it turns out) notion is as follows.

Definition. An (unbounded) positive operator affiliated to the algebra $R(\pi)$ is said to be semi-invariant of degree $\lambda$ if $\pi(g)^{-1} D \pi(g)=$ $\lambda(g) D$ where $\lambda$ is as usual a homomorphism of $G$ into $R^{+}$.

The relation between these two notions is made clear by the following result which also makes precise our statement in the first section that $\pi$ can have an operator as its formal degree (if and) only if $\pi$ is semi-finite.

THEOREM 2. Let $\pi$ be primary and $\phi$ a semi-invariant weight of degree $\lambda$ for $\pi$. Then there exists a positive operator $D$ affiliated to $R(\pi)$, semi-invariant of degree $\lambda$, if and only if $R(\pi)$ is semi-finite. In this case $\phi(x)=\operatorname{Tr}\left(D^{1 / 2} x D^{1 / 2}\right)($ or $\phi=\operatorname{Tr}(D \cdot)$ in the language of [3]) where $\operatorname{Tr}$ is a trace on $R(\pi)$.

Proof. If there exists such a $D$, then $u_{t}=D^{i t}$ is a unitary group in $R(\pi)$. Moreover, $\pi(g) u_{t} \pi(g)^{-1}=\lambda(g)^{-i t} u_{t}$ by semi-invariance; we then have $u_{t} \pi(g) u_{t}^{*}=\pi(g) \lambda(g)^{i t}$. Now if $\sigma_{t}^{\phi}$ is the modular automorphism group associated to $\phi, \sigma_{t}^{\phi}=\sigma_{t}^{a \phi}$ for any $a>0$, and by the properties of Radon-Nikodym derivatives, $\quad \pi(g)^{*} \sigma_{t}^{\phi}(\pi(g))=\left(D \phi^{\pi(g)}: D \phi\right)_{t}=$ $\exp (+i t \log (\lambda(g))) 1$. After rearranging this, we see that $\sigma_{t}^{\phi}(\pi(g))=$ $\pi(g) \lambda(g)^{i t}$, and hence that $\sigma_{t}^{\phi}$ agrees on a set of generators of $R(\pi)$ with the inner automorphism defined by $u_{t}$. Thus $\sigma_{t}^{\phi}$ is in fact inner and consequently $R(\pi)$ is semi-finite.

In this case $\operatorname{Tr}\left(D^{1 / 2} \cdot D^{1 / 2}\right)=\operatorname{Tr}(D \cdot)$ in the language of [3] evidently 
defines a semi-finite weight which one sees at once is semi-invariant of degree $\lambda$, and hence by Theorem 1 is a multiple of $\phi$.

Conversely if $R(\pi)$ is semi-finite, let $\operatorname{Tr}$ be a trace. Then the Radon-Nikodym derivative $(D \phi: D \mathrm{Tr})_{t}$ is a one-parameter group in $R(\pi)$ and is therefore of the form $D^{\text {it }}$ with $D$ affiliated to $R(\pi)$. By the same kind of calculations one sees immediately that $D$ is semi-invariant of degree $\lambda$ and this completes the proof.

(3) If $\phi$ is a semi-invariant weight on $R(\pi)$ we introduce the space $n_{\phi}=\left\{x: \phi\left(x^{*} x\right)<\infty\right\}$. This is a left ideal and is two-sided invariant under multiplication by $\pi(g)$. It is also $\sigma$-weak dense in $R(\pi)$ as this is part of the definition of a semi-finite weight. We let $m_{\phi}$ be the set of linear combinations of elements of the form $y^{*} x, x, y \in n_{\phi}$. Evidently $m_{\phi}$ is also closed under left and right multiplication by $\pi(g)$. It is also well known that there is a unique linear functional on $m_{\phi}$ denoted again by $\phi$ which agrees with the original weight on elements of the form $x^{*} x$, $x \in n_{\phi}$.

Since $\phi$ is faithful, $n_{\phi}$ is a pre-Hilbert space, and we let $H_{\phi}$ be its completion and let $\Delta$ and $J$ be the modular operator and the unitary conjugation defined by the weight $\phi$ via Tomita-Takesaki theory. We define the left representation of $G$ by $L(g) a=\pi(g) a$ for $a \in n_{\phi}$. Clearly $L(g)$ extends to a unitary operator on $H_{\phi}$ and $L$ is a unitary representation of $G$ on $H_{\phi}$ which generates the von Neumann algebra $M$ (isomorphic to $R(\pi)$ ) of left multiplications on $H_{\phi}$. If we define $R(g)(a)=\lambda(g)^{-1 / 2} a \pi(g)^{-1}$ for $a \in n_{\phi}$ it is easy to see that $R(g)$ extends to a unitary operator on $H_{\phi}$.

Now if $x \in n_{\phi} \cap n_{\phi}^{*}$, it follows that $x \pi(g) \in n_{\phi} \cap n_{\phi}^{*}$ since as we have noted $n_{\phi}$ is left and right invariant under $\pi(g)$. Now if $S(x)=x^{*}$ for such elements, we see that $(L(g) S)(x)=\lambda(g)^{1 / 2} S R(g)(x)$ and hence $L(g) S=\lambda(g)^{1 / 2} S R(g)$ where $S$ is the closure of the map $x \rightarrow x^{*}$ on $n_{\phi} \cap n_{\phi}^{*}$. It also follows that $R(g)$ and $L(g)$ send the domain of $S$ into itself, and as $S=J \Delta^{1 / 2}$ is the polar decomposition of $S$, we see that $R(g)$ and $L(g)$ send the domain of $\Delta^{1 / 2}$, which is the domain of $S$, into itself. Moreover

$$
\begin{aligned}
L(g) J \Delta^{1 / 2} & =\lambda(g)^{1 / 2} J \Delta^{1 / 2} R(g) \\
& =J R(g) \lambda(g)^{1 / 2} R(g)^{*} \Delta^{1 / 2} R(g)
\end{aligned}
$$

and by the unicity of the polar decomposition we have $L(g) J=J R(g)$ or $J L(g) J=R(g)$ and $\Delta^{1 / 2}=\lambda(g)^{1 / 2} R(g)^{*} \Delta^{1 / 2} R(g), \quad$ or equivalently $L(g) \Delta^{1 / 2} L(g)^{-1}=\lambda(g)^{-1 / 2} \Delta^{1 / 2}$.

The formula $J L(g) J=R(g)$ shows that $R(g)$ is a unitary representation which generates the commutant $M^{\prime}=J M J$ of the von Neumann 
algebra generated by $L$. It is also evidently the contragredient representation to $R$.

In the sequel we shall need the following fact about stability of $n_{\phi}$ and $m_{\phi}$. Let $a$ be a continuous function of compact support on $G$ and let $A=\int a(s) \pi(s) d s$ be the corresponding operator in $R(\pi)$ where $d s$ is left Haar measure.

Proposition 3.1. If $x \in n_{\phi}, A x$ and $x A \in n_{\phi}$; if $y \in m_{\phi}, A y$ and $y A \in m_{\phi}$ and $\phi(A y)=\int a(s) \phi(\pi(s) y) d s$, and similarly for $\phi(y A)$.

Proof. It is immediate that if $x \in n_{\phi}$ then $B x \in n_{\phi}$ for any $B \in$ $R(\pi)$. Now if $A$ has the special form as in the statement, we may find a sequence of operators $B_{n}$ each of which is a finite linear combination of the operators $\pi(s), s \in G$ such that $\left|B_{n}\right|$ is dominated by the $L_{1}$ norm of $a$ and so that $B_{n} \rightarrow A{ }^{*}$-strongly. We simply use "Riemann sums" to approximate the integral defining $A$. Then $\left(x B_{n}\right)^{*}\left(x B_{n}\right)=B_{n}^{*} x{ }^{*} x B_{n}$ evidently converges weakly, and hence $\sigma$-weakly since $\left|B_{n}\right| \leqq K$, to $A x^{*} x A$. Since $\phi$ is $\sigma$-weak lower semi-continuous, it follows that

$$
\phi\left((x A)^{*} x A\right)=\phi\left(A x^{*} x A\right) \leqq \lim \phi\left(\left(x B_{n}\right)^{*}\left(x B_{n}\right)\right)=\lim \left|x B_{n}\right|^{2}
$$

norms taken in $H_{\phi} . \quad$ But now if $a(s)=\lambda^{1 / 2}(s) \Delta\left(s^{-1}\right) \tilde{a}\left(s^{-1}\right)$ where $\Delta$ is the usual modular function on $G$, it follows that when we integrate the right representation $R$ against the function $\tilde{a}, R(\tilde{a}) x=\int \tilde{a}(s) R(s) x d s$ converges as a Bochner integral in $H_{\phi}$. On the other hand, it is evident that $x B_{n}$ are Riemann sums approximating this integral so that $x B_{n} \rightarrow R(\tilde{a}) x$. Thus $\lim \left|x B_{n}\right|^{2}=|R(\tilde{a}) x|^{2}$ and so $\phi\left((x A)^{*}(x A)\right) \leqq$ $|R(\tilde{a}) x|^{2}$ and $x A \in n_{\phi}$ as desired.

Moreover, by the same reasoning, for any $m$

$$
\phi\left(\left(x\left(A-B_{m}\right)\right)^{*} x\left(A-B_{m}\right)\right) \leqq \lim _{n}\left|x\left(B_{n}-B_{m}\right)\right|^{2},
$$

and since $x B_{n}$ is Cauchy in $H_{\phi}$ it follows that $\left|x\left(A-B_{m}\right)\right|^{2} \rightarrow 0$ in $n_{\phi}$ and hence that $x A=R(\tilde{a}) x$. Thus for any $z \in n_{\phi},(x A, z)=(R(\tilde{a}) x, z)$, which means using the Bochner integral for $R(\tilde{a}) x$ that

$$
\begin{aligned}
\phi\left(z^{*} x A\right) & =\int \phi\left(z^{*} x \pi(s)^{-1}\right) \lambda(s)^{-1 / 2} \tilde{a}(s) d s \\
& =\int \phi\left(z^{*} x \pi(s)\right) a(s) d s .
\end{aligned}
$$


Now since any element of $m_{\phi}$ can be written as a linear combination of elements of the form $z^{*} x$, we have established that $y A \in m_{\phi}$ if $y \in m_{\phi}$ and that the desired integral formula holds. The result for $A y$ follows by the same reasoning.

We now introduce the notion of square integrability and establish equivalent criteria. There are several different notions of matrix coefficients and we first examine these possibilities. We first observe that a "geometric" matrix coefficient $(x, \pi(g) y)=\left(\pi(g)^{-1} x, y\right)$ of a representation $\pi$ can be written in the form $f\left(\pi(g)^{-1}\right)$ where $f(a)=(a(x), y)$ is an element of the predual $R(\pi)_{*}$ of $R(\pi)$. In fact, it is clear that any $f \in R(\pi)_{*}$ is the limit in norm of finite linear combinations of $f$ 's of the above form. Hence the most general "matrix coefficient" of $\pi$ is a function of the form $f\left(\pi(g)^{-1}\right)$ for some $f$ in the predual of $R(\pi)$. Now suppose that $\phi$ is a semi-invariant weight on $R(\pi)$ and that $x \in$ $m_{\phi}$. Then $\pi(g)^{-1} x \in m_{\phi}$ so that $\phi\left(\pi(g)^{-1} x\right)$ is well defined and could also with much justification be called a matrix coefficient of $\pi$. We denote these matrix coefficients as $m_{x}$ with $\phi$ to be understood. It should be noted that the map $z \rightarrow \phi(z x)$ may not be everywhere defined and hence not in the predual so that this matrix coefficient may not be of the above form.

We fix once and for all a left Haar measure $\mu_{l}$ on $G$ and denote by $\Delta$ the modular function. Then $\mu_{r}$ defined by $d \mu_{r}(x)=\Delta(x)^{-1} d \mu_{l}(x)$ is a right Haar measure.

Definition. A primary representation $\pi$ is square integrable if $R(\pi)$ has a semi-invariant weight $\phi$ of degree $\Delta$ so that for some $x \in m_{\phi}$, $m_{x}(g)=\phi\left(\pi(g)^{-1} x\right)$ is a square integrable function on $G$ with respect to $u_{l}$.

We first note the following fact.

Proposition 3.2. If $\pi$ is square integrable, then the set $s_{\phi}$ of $x \in$ $R(\pi)$ such that $m_{x} \in L^{2}\left(\mu_{l}\right)$ is $\sigma$-strong dense in $R(\pi)$, and $s_{\phi} \subset m_{\phi} \subset n_{\phi} \subset$ $H_{\phi}$ is dense in the Hilbert space $H_{\phi}$.

Proof. Note that $s_{\phi}$ is closed under multiplication by $\pi(g)$ on both sides as $\phi\left(\pi(g)^{-1} \pi(h) x \pi(k)\right)=\Delta(k)^{-1} \phi\left(\pi\left(h^{-1} g k^{-1}\right)^{-1} x\right)$ which is square integrable in $g$ if $m_{x}$ is. It follows that the $\sigma$-strong closure of $s_{\phi}$ has the same property and hence is a two-sided ideal, which is necessarily $R(\pi)$ as $\pi$ is primary. The second statement follows in the same way.

We shall subsequently show that if some $m_{x}$ is square integrable, 
then $m_{x}$ is square integrable for all $x \in m_{\phi}$. We note of course that this notion of square integrability is invariant under quasi-equivalence as quasi-equivalence of $\pi_{1}$ and $\pi_{2}$ simply means that $R\left(\pi_{1}\right)$ and $R\left(\pi_{2}\right)$ are isomorphic by an isomorphism earrying $\pi_{1}(g)$ onto $\pi_{2}(g)$. We shall now show that the various other possible definitions of square integrability all coincide.

THEOREM 3. For a primary representation $\pi$ the following are equivalent :

(1) $\pi$ is square integrable;

(2) $(x, \pi(\cdot) y) \in L^{2}\left(\mu_{l}\right)$ and is non-zero for some $x, y$;

(3) $\exists f \in R(\pi)_{*}, f\left(\pi(\cdot)^{-1}\right) \in L^{2}\left(\mu_{l}\right), f \neq 0$;

(4) $\pi$ is quasi-equivalent to a summand of the left regular representation.

Proof. We note that Rosenberg [6] has established the equivalence of (2) and (4). We have already noted that $(2) \Rightarrow(3)$ above. To see that (3) $\Rightarrow(4)$, let $\sigma=\infty \pi$, and then using the standard form for elements in the predual of $R(\pi)$, it is clear that $f\left(\pi(g)^{-1}\right)$ is an ordinary matrix coefficient of $\sigma$. Hence by Rosenberg's result, $\sigma$ and hence $\pi$, is quasi-equivalent to a subrepresentation of the regular representation. Thus (2), (3) and (4) are equivalent and we shall complete the argument by establishing the equivalence of (1) and (4).

Suppose that (4) holds; then as (1) is invariant under quasiequivalence, we can assume that $\pi$ is a summand of the left regular representation which is invariant under left and right translations. If $M$ is the von Neumann algebra generated by the left regular representation, we may then identify $R(\pi)$ as the weakly closed two-sided ideal in $M$ determined by the projection onto the subspace $H(\pi)$ of $L^{2}\left(\mu_{l}\right)$ defined by $\pi$.

Now $M$ possesses a distinguished weight $\phi$, defined by evaluation at the identity [8]. More precisely, if $h$ is continuous with compact support on $G$ and if $f=h^{*} h$, then $f$ is continuous and $f(e)=|h|_{2}^{2}$, the square of the $L^{2}$ norm of $h$. If $L_{f}=\pi(f)$ is the corresponding operator of left convolution by $f, L_{f} \in M$ and one defines $\phi\left(L_{f}\right)=f(e)$. Using Tomita theory, this extends to a normal weight $\phi$ on $M$. An easy verification shows that $\phi\left(\pi(g) L_{f} \pi(g)^{-1}\right)=\Delta(g) \phi\left(L_{f}\right)$ and by extension the same is true for the weight on $M$ and so $\phi$ is semi-invariant of degree $\Delta$ and faithful.

We restrict $\phi$ to $R(\pi)$ and it is again semi-finite and semi-invariant of degree $\Delta$. It remains to find an $x$ so that $m_{x}$ is square integrable. Now if $f=h^{*} h$ as above, one verifies by direct calculation that $L_{f} \in m_{\phi}$ and $\phi\left(\pi(s)^{-1} L_{f}\right)=f(s)$. Let $P$ be the projection of $L_{2}(G)$ 
onto $H(\pi)$; then $P$ is in the center of $M$ and $R(\pi)=P M$. Since $P L_{f} \leqq L_{f}$ and $P L_{f}=P L_{h}^{*} P L_{h}$, it follows that $P L_{f}$ is in $m_{\phi}$ and we claim that $\phi\left(\pi(s)^{-1} P L_{f}\right)=(P f)(s)$, where $f$ is viewed on the right hand side as a vector in $L^{2}(G)$. In fact, let $a$ be a continuous function with compact support on $G$ and let $A=\int \pi(s)^{-1} a(s) d s \in M$. Then evidently

$$
\begin{gathered}
\int a(s) \phi\left(\pi(s)^{-1} \pi(t) L_{f}\right) d s=\phi\left(A \pi(t) L_{f}\right) \\
=\int a(s) f\left(t^{-1} s\right) d s=(\pi(t) f, \bar{a})
\end{gathered}
$$

where in the last term $a$ is viewed as an element of $L^{2}(G)$. Now by the general theory of weights, $A x L_{f} \in m_{\phi}$ for $x \in M$ and $x \rightarrow \phi\left(A x L_{f}\right)$ is in the predual. Evidently the same is true for $x \rightarrow(x f, \bar{a})$ where $x f$ is the result of applying the operator $x \in M$ to the vector $f \in L^{2}(G)$. The above identity asserts equality of these linear functionals for $x$ of the form $\pi(t), t \in G$, and it follows that they are equal for all $x \in M$ and in particular for $x=P$. Then we have $\phi\left(A P L_{f}\right)=(P f, \bar{a})$, but since $P L_{f} \in m_{\phi}$, it follows from Proposition 3.1 that the left side above can be written as an integral over $G$ so that we have

$$
\int a(s) \phi\left(\pi(s)^{-1} P L_{f}\right) d s=\int a(s) P f(s) d s .
$$

Since this holds for all $a^{*}$ which are continuous and compactly supported, it follows that $\phi\left(\pi(s)^{-1} P L_{f}\right)=P f(s)$ a.e. and therefore that $\phi\left(\pi(s)^{-1} P L_{f}\right)$ is a square integrable function as desired.

We now turn to the converse (1) $\Rightarrow(4)$, so suppose that $\phi$ is a semi-invariant weight of degree $\Delta$ for $R(\pi)$ where $\pi$ is a primary representation, and let $n_{\phi}, m_{\phi}, H=H_{\phi}$ (the completion of $n_{\phi}$ ) $\Delta, J, L(g), R(g)$ be as above and suppose that $m_{x}(g)=\phi\left(\pi(g)^{-1} x\right)$ is in $L_{2}\left(\mu_{l}\right)$ for some $x \in m_{\phi}$. Let $x=\Sigma y_{i}^{*} z_{i}$ with $y_{i}, z_{l} \in n_{\phi}$. Now let $H^{n}$ be the $n$-fold direct sum of $H$ with itself and let $R^{n}$ be the $n$-fold direct sum of $R$ acting on $H^{n}$. Then $y=\left(y_{1}, \cdots, y_{n}\right)$ and $z=\left(z_{1}, \cdots, z_{n}\right)$ are elements of $H^{n}$ and the matrix coefficient $\left(z, R^{n}(g) y\right)$ is equal to $\Delta(g)^{-1 / 2} m_{x}\left(g^{-1}\right)$. But since $m_{x}(\cdot)$ is assumed to be square integrable, it follows now that $\left(z, R^{n}(\cdot) y\right)$ is also square integrable. Then by Rosenberg's result, $R^{n}$ is quasi-contained in the regular representation and hence so is $R$. But since $R$ and $L$ are contragredients, and $L$ is quasi-equivalent to $\pi$, and as the regular representation is selfcontragredient, $\pi$ is quasi-contained in the regular representation and this is (4). 
Now that this result is established we can proceed to establish the orthogonality relations for the "matrix coefficients" $m_{x}$. Introduce as above the space $H(\pi)$, the sum of all left invariant subspaces of $L^{2}\left(\mu_{l}\right)$ which are quasi-equivalent to $\pi$.

THEOREM 4. If $\pi$ is square integrable, then $m_{x} \in L^{2}\left(\mu_{l}\right)$ for all $x \in m_{\phi}$ and $\phi$ may (uniquely) be normalized so that $x \rightarrow m_{x}$ extends to unitary operator from $H_{\phi}$ onto $H(\pi)$. In particular, we have orthogonality relations

$$
\int m_{x}(g) \overline{m_{y}(g)} d \mu_{l}(g)=\phi\left(y^{*} x\right)
$$

for $x, y \in m_{\phi}$.

Proof. As before, let $s_{\phi}=\left\{x: x \in m_{\phi}, m_{x} \in L^{2}\left(\mu_{l}\right)\right\}$; then easy computations show that $m_{L(h) x}(g)=m_{x}\left(h^{-1} g\right)$ and $m_{R(h) x}(g)=$ $m_{x}(g h) \Delta(h)^{1 / 2}$. Thus $m$ is a densely defined linear operator from $s_{\phi} \subset m_{\phi} \subset n_{\phi} \subset H_{\phi}$ into $L^{2}\left(\mu_{l}\right)$ which intertwines the two-sided representation $L \times R$ of $G \times G$ on $H_{\phi}$ with the two-sided regular representation $U$ of $G \times G$ on $L^{2}\left(\mu_{l}\right)$ given by $\left(U\left(t_{1}, t_{2}\right) f\right)(s)=f\left(t_{1}^{-1}, s t_{2}\right) \Delta\left(t_{2}\right)^{1 / 2}$. Now by Theorem 3 we know that $\pi$ is quasi-contained in the regular representation and hence that the von Neumann algebra $R(\pi)$ is isomorphic to a direct summand of the von Neumann algebra generated by the left regular representation. Therefore, by the unicity of semiinvariant weights and the argument of the first part of the proof of Theorem 3, we know that if $L_{f}$ is left convolution by $f$, a continuous function on $G$ with compact support, and if $e$ is the unit in $R(\pi)$ viewed as a central projection in the von Neumann algebra of the left regular representation, that $e L_{f}=L_{f} e=e L_{f} e$ is in $n_{\phi}$ and that $\phi\left(\left(e L_{f}\right)^{*} e L_{f}\right) \leqq$ $f^{*} f(1)$ where 1 is the identity element in $G$. But under the quasiequivalence of $\pi$ with a summand of $L_{2}\left(\mu_{l}\right)$, the operator $e L_{f}$ is nothing other than $T(f)=\int f(s) \pi(s) d \mu_{l}(s)$, and so $T(f) \in n_{\phi}$. Also note that $T(f)^{*} \in n_{\phi}$. Moreover, for any $x \in m_{\phi}$,

$$
\phi\left(T(f)^{*} x\right)=\phi\left(\left(\int \overline{f(s)} \pi(s)^{-1} d g\right) x\right)=\int m_{x}(s) \bar{f}(s) d s .
$$

If we read this in terms of inner products this says that $f \rightarrow T(f)$ is the adjoint of the operator $x \rightarrow m_{x}$. Thus $m$ defined on $s_{\phi}$ has a densely defined adjoint and so is closeable. But now $m$ intertwines $L \times R$ on $H_{\phi}$ with $U$ on $L^{2}\left(\mu_{l}\right)$. Now $L \times R$ is irreducible on $H_{\phi}$, and it follows 
since $m$ is closeable that $m$ is a multiple of an isometry and hence after proper normalization of $\phi$ extends to a unitary intertwining operator of $H_{\phi}$ into $L^{2}\left(\mu_{l}\right)$. Evidently its range is contained in $H(\pi)$ and since $H(\pi)$ is irreducible under $U$, its range is all of $H(\pi)$. This completes the proof except for the statement that $s_{\phi}$ is all of $m_{\phi}$.

To see this, let $x \in m_{\phi}$ and choose a sequence $x(n) \in s_{\phi}$ with $x(n) \rightarrow x$ in $H_{\phi}$. Then $m_{x(n)}(\cdot)=\phi\left(\pi(\cdot)^{-1} x(n)\right)$ converges to a limit $q$ in $L^{2}\left(\mu_{l}\right)$. But now as above, if $f$ is continuous and compactly supported on $G, T(f) \in n_{\phi}$ and therefore $\phi\left(T(f)^{*} x(n)\right)$ converges to $\phi\left(T(f)^{*} x\right)$. As above, this means that

$$
\int \bar{f}(s) m_{x(n)}(s) d \mu_{l} \rightarrow \int \bar{f}(s) \phi\left(\pi(s)^{-1} x\right) d \mu_{l}
$$

and it follows that $q(s)=\phi\left(\pi(s)^{-1} x\right)$ a.e. so that $\phi\left(\pi(\cdot)^{-1} x\right)$ is square integrable and hence $x \in s_{\phi}$.

REMARK. As one can see from the above arguments in Theorems 3 and 4 , the objects $m_{x}\left(x \in m_{\phi}\right)$ that we have called matrix coefficients are really matrix coefficients of the right representation $R$ which is the contragradient of $L$ or, equivalently, $\pi$. The matrix coefficients of $L$ or $\pi$ are simply the complex conjugates of those of $R$, specifically $(L(g) u, v)=(R(g) J u, J v)$ for $u, v \in H_{\phi}$. As an alternative to the $m_{x}$ 's one could consider instead functions of the form $\phi\left(a^{*} \pi(g) b\right), a, b \in n_{\phi}$ which by a simple calculation becomes $\Delta(g)^{-1 / 2} \phi\left((J a)^{*}(J b) \pi(g)^{-1}\right)=$ $\Delta(g)^{1 / 2} \bar{m}_{c}(g)$ where $c=(J a)^{*} J b$ provided, of course, that $J a$ and $J b$ are in $n_{\phi}$. We have chosen the $m_{x}$ as our basic objects since formulas come out more elegantly this way.

Definition. If $\pi$ is a square integrable representation we shall call the semi-invariant weight $\phi$ normalized as in Theorem 4 the formal degree of $\pi$. Thus we see that the formal degree which classically was a positive number becomes first a semi-invariant positive operator as in [2] and now even more generally becomes a semi-invariant weight on $R(\pi)$.

(4) We now turn to the case when $R(\pi)$ is semi-finite so that the semi-invariant weight $\phi$ is of the form $\phi(x)=\operatorname{Tr}\left(D^{1 / 2} x D^{1 / 2}\right)$ where $\operatorname{Tr}$ is a trace on $R(\pi)$ and $D^{1 / 2}$ a uniquely determined semi-invariant positive operator affiliated to $R(\pi)$. We let $L^{1}(\operatorname{Tr})$ and $L^{2}(\operatorname{Tr})$ be the spaces of integrable and square integrable operators relative to the trace [7]. The natural action of $R(\pi)$ on $L^{2}(\mathrm{Tr})$ by left multiplication is of course the GNS construction applied to the weight $\mathrm{Tr}$, and we so realize $R(\pi)$. Then $D$ and all its powers can be realized as self-adjoint 
operators on $L^{2}(\mathrm{Tr})$. Of especial importance to us will be the (dense) domain of definition of the operator $D^{-1 / 2}, \operatorname{dom}\left(D^{-1 / 2}\right)$. Now let $p=$ $\left\{x, x \in R(\pi) \cap L^{2}(\operatorname{Tr})\right.$, range $\left.\left(x^{*}\right) \subset \operatorname{dom}\left(D^{-1 / 2}\right)\right\}$, where range $\left(x^{*}\right)$ is the range of $x^{*}$ as an operator on $L^{2}(\operatorname{Tr})$, and let $q$ be the span of elements of the form $y^{*} x, x, y \in p$. Note that $p$ and $q$ are linear spaces invariant under right and left multiplication by $\pi(g), g \in G$. Now if $x \in p$, the closed operator $D^{-1 / 2} x^{*}$ is everywhere defined, hence bounded and so $x D^{-1 / 2}$ is bounded on its domain and hence has a continuous extension to a bounded operator $f(x)$ which is necessarily in $R(\pi)$. Evidently $\phi\left(f(x)^{*} f(x)\right)=\operatorname{Tr}\left(x^{*} x\right)$ so $f(x) \in n_{\phi}$ and $f$ is an isometry. In the same way, the map $l$ defined on $q$ by $l\left(y^{*} x\right)=\left(D^{-1 / 2} y^{*} x D^{-1 / 2}\right)^{-}$is well defined and sends $q$ into $m_{\phi}$. Finally, note that $q=q^{*}$ and that if $u \in q$, then $D^{-1 / 2} u$ is well defined and is bounded and is square integrable relative to the trace, for if $u=y^{*} x, x, y \in p$, then $D^{-1 / 2} u=\left(D^{-1 / 2} y^{*}\right)(x)$. Since $D^{-1 / 2} y^{*}$ is bounded and $x$ is square integrable and bounded, the product is bounded and square integrable as asserted.

In the context of a semi-finite primary representation, we are also interested in functions $n_{u}(g)=\operatorname{Tr}\left(\pi(g)^{-1} u\right)$ which also have a claim to be called matrix coefficients of $\pi$. It is evident that $n_{u}$ is well defined and is a continuous function on $G$ for any $u \in L^{1}(\mathrm{Tr})$. We begin the study of such matrix coefficients by considering the case $u \in q \subset L^{1}(\mathrm{Tr})$. We have the following result.

THEOREM 5. If $u \in q=q^{*}, n_{u}$ is square integrable with respect to left Haar measure and if $u, v \in q$,

$$
\begin{aligned}
\int n_{u}(g) \overline{n_{v}(g)} d \mu_{l} & =\operatorname{Tr}\left(\left(D^{-1 / 2} u^{*}\right)^{*} D^{-1 / 2} v^{*}\right) \\
& =\left(D^{-1 / 2} v^{*}, D^{-1 / 2} u^{*}\right)_{L^{2}(\mathrm{Tr})}
\end{aligned}
$$

Proof. This is simply a matter of rephrasing Theorem 4 and we omit the details.

The above result leaves unanswered the question of providing necessary and sufficient conditions for $n_{u}$ to be square integrable and we turn to this question now. Let

$$
S=\left\{u: u \in L^{1}(\operatorname{Tr}) \cap L^{2}(\operatorname{Tr}), n_{u} \in L^{2}\left(\mu_{l}\right)\right\}
$$

Then we have the following result.

Theorem 6. Let $u \in L^{2}(\mathrm{Tr})$; then $u \in S \Leftrightarrow u$ (equivalently 
$\left.u^{*}\right) \in L^{1}(\operatorname{Tr}), \quad$ and $u^{*} \in \operatorname{dom}\left(D^{-1 / 2}\right)$. If $u, v \in S$, then $\left(n_{u}, n_{v}\right)=$ $\left(D^{-1 / 2} v^{*}, D^{-1 / 2} u^{*}\right)$.

Proof. We define the operator $N$ on $S$ into $L_{2}(G)$ by $N(u)=$ $n_{u}$. The set $S$ is dense in $L^{2}(\operatorname{Tr})$ as $q$ is and is two-sided invariant under $\pi(g)$. Moreover, if $\rho\left(t_{1}, t_{2}\right) u=\pi\left(t_{1}\right) u \pi\left(t_{2}\right)^{-1}$ for $u \in L^{2}(\operatorname{Tr})$ and $\left(u\left(t_{1}, t_{2}\right) f\right)(g)=f\left(t_{1}^{-1} g t_{2}\right) \Delta\left(t_{2}\right)^{1 / 2}$ is as before, it is clear that

$$
N \rho\left(t_{1}, t_{2}\right) u=\Delta\left(t_{2}\right)^{-1 / 2} U\left(t_{1}, t_{2}\right) N(u) \text { for } u \in S
$$

Now $N(u)$ is immediately seen to be a matrix coefficient of the representation $\sigma$ of $G$ on $L_{2}(\mathrm{Tr})$ given by left multiplication by $\pi$. But $\sigma$ is quasi-equivalent to $\pi$, and it follows by Rosenberg's reasoning [6] that $N(u) \in H(\pi) \subset L^{2}\left(\mu_{l}\right)$.

Now $R(\pi)$ can be realized as a von Neumann algebra operating on $H(\pi)$ so that $\pi(g) \in R(\pi)$ is left translation by $g^{-1}$. Let $b \rightarrow \lambda(b)$ be this realization, $b \in R(\pi)$. Then the formulas in the previous paragraph say that $N(b u)=\lambda(b) N(u)$ for $u \in S$, and $b$ a finite linear combination of the operators $\pi(g), g \in G$. But now if $b_{n}$ is a sequence of such linear combinations converging to a limit $b \in R(\pi), \sigma$ strongly, and with $\left|b_{n}\right| \leqq k<\infty$, then $N\left(b_{n} u\right)(g)=\operatorname{Tr}\left(\pi(g)^{-1} b_{n} u\right)$ converges to $\operatorname{Tr}\left((g)^{-1} b u\right)$ for each $g \in G$. But $\lambda\left(b_{n}\right) N(u)$ converges to $\lambda(b) N(u)$ as $\lambda$ is normal, and therefore $\operatorname{Tr}\left(\pi(g)^{-1} b u\right)=(\lambda(b) u)(g)$ is in $L^{2}\left(\mu_{l}\right)$ as a function of $g$. But $b u \in L^{1}(\mathrm{Tr})$ and so $b u \in S$ and $N(b u)=\lambda(b) N(u)$.

Now let $u_{n} \in S$ and $u_{n} \rightarrow u$ in $L^{2}(\operatorname{Tr})$, and suppose $N\left(u_{n}\right)$ has a limit $v$ in $H(\pi)$. Then for any $b \in L^{2}(\operatorname{Tr}) \cap R(\pi), b u_{n} \in L^{1}(\operatorname{Tr})$ and $b u_{n} \rightarrow b u$ in $L^{1}(\operatorname{Tr})$ and so $N\left(b u_{n}\right)(g) \rightarrow \operatorname{Tr}\left(\pi(g)^{-1} b u\right)$ for each $g \in$ G. But $N\left(b u_{n}\right)=\lambda(b) N\left(u_{n}\right)$ converges to $\lambda(b) v$ in $H(\pi)$. But this says that $\lambda(b) v$ is determined by $u$ alone for each $b \in L^{2}(\operatorname{Tr}) \cap R(\pi)$, and that $u$ determines $v$ uniquely. This says of course that $N$ is closeable as a densely defined operator $S \rightarrow H(\pi)$; and if $\bar{N}$ is its closure, we have by continuity $\bar{N} \rho\left(t_{1}, t_{2}\right)=\Delta\left(t_{2}\right)^{-1 / 2} U\left(t_{1}, t_{2}\right) \bar{N}$.

We then write the polar decomposition $\bar{N}=W K$ where the domain of $K$ is the domain of $N$, and where $W$ is a unitary equivalence between the (irreducible) representations $\rho$ and $U$, and where $K \rho\left(t_{1}, t_{2}\right)=$ $\Delta\left(t_{2}\right)^{-1 / 2} \rho\left(t_{1}, t_{2}\right) K$. Then $K$ as an operator on $L^{2}(\operatorname{Tr})$ is affiliated to $R(\pi)^{\prime}$ since it commutes with $\pi(g)$ operating as left multiplications, and is semi-invariant under right multiplications. It follows that $J K J$ is an operator affiliated to $R(\pi)$ and is semi-invariant of degree $\Delta^{1 / 2}$. Therefore it is precisely $D^{-1 / 2}$ up to a multiple as this is the only semi-invariant operator of this degree (Theorems 1 and 2). Thus up to scalars $K(u)=\left(D^{-1 / 2} u^{*}\right)^{*}$ and the domain of $K$, which is the domain of $\bar{N}$ as already noted, is precisely the set of $u$ such that $u^{*}$ is in the domain of 
$D^{-1 / 2}$. We know now that $S^{*} \subset L^{1}(\operatorname{Tr}) \cap \operatorname{dom}\left(D^{-1 / 2}\right)$ and that the orthogonality relations hold up to a scalar as $W$ is unitary; that the normalization is correct so that $K=D^{-1 / 2}$ follows from Theorem 5 .

It remains to show that $S^{*}=L^{1}(\operatorname{Tr}) \cap \operatorname{dom}\left(D^{-1 / 2}\right)$. If $u$ is in the right hand side, $\bar{N}(u)$ is well defined and it follows as above that $\lambda(b) \bar{N}(u)(g)=\operatorname{Tr}\left(\pi(g)^{-1} b u\right)$ for $b \in L^{2}(\operatorname{Tr}) \cap R(\pi)$. Now let $b_{n}$ be a bounded sequence with $b_{n} \rightarrow 1 \quad \sigma$-strong with $b_{n} \in L^{2}(\mathrm{Tr})$. Then $\lambda\left(b_{n}\right) \bar{N}(u) \rightarrow \bar{N}(u)$ and $\operatorname{Tr}\left(\pi(g)^{-1} b_{n} u\right) \rightarrow \operatorname{Tr}\left(\pi(g)^{-1} u\right)$ for each $g$ as $u \in L^{1}(\operatorname{Tr})$. Thus $N(u)(g)=\operatorname{Tr}\left(\pi(g)^{-1} u\right)$ a.e., and this says that $u \in S$ as desired. This completes the proof.

We can reformulate Theorem 6 in terms of right Haar measure as follows (proof omitted).

Theorem 7. Let $u \in L^{2}(\mathrm{Tr}) \cap L^{1}(\mathrm{Tr})$. Then $n_{u} \in L^{2}\left(\mu_{r}\right)$ iff $u \in$ $\operatorname{dom}\left(D^{-1 / 2}\right)$ and then for such $u$ and $v$

$$
\int n_{u}(g) \overline{n_{v}(g)} d \mu_{r}(g)=\left(D^{-1 / 2} u, D^{-1 / 2} v\right)
$$

In conclusion it is instructive to see that we recapture the necessary and sufficient condition in [2] and [4] for square integrability of the ordinary matrix coefficients of an irreducible square integrable representation. Let $\pi$ be such and let $x, y$ be vectors in the Hilbert space $H$ of $\pi$, and let $c_{x, y}=(x, \pi(\cdot) y)$. Then $L^{2}(\mathrm{Tr})$ is of course the Hilbert-Schmidt operators and we let $T(x, y)(w)=(w, y) x$ be the rank one operator associated to $x$ and $y$. A simple calculation shows that $c_{x, y}=n_{T(x, y)}$ in the notation of Theorems 5 and 6 . Now $T(x, y)^{*}=T(y, x)$ and $T(y, x) \in \operatorname{dom}\left(D^{-1 / 2}\right)$ (viewing $D^{-1 / 2}$ as an operator on $L^{2}(T r)$ ) iff $y \in \operatorname{dom}\left(D^{-1 / 2}\right)$ with $D^{-1 / 2}$ viewed here as an operator on $H$; if this is the case, $D^{-1 / 2} T(y, x)=T\left(D^{-1 / 2} y, x\right)$. Thus Theorem 6 implies that $c_{x, y}$ is square integrable with respect to left Haar measure iff $y \in \operatorname{dom}\left(D^{-1 / 2}\right) \subset$ $H$ and if $y^{\prime}, y \in \operatorname{dom}\left(D^{-1 / 2}\right)$, then

$$
\begin{aligned}
\left\langle c_{x, y}, c_{x^{\prime}, y^{\prime}}\right\rangle & =\left\langle T\left(D^{-1 / 2} y^{\prime}, x^{\prime}\right), T\left(D^{-1 / 2} y, x\right)\right\rangle \\
& =\operatorname{Tr}\left(T\left(x, D^{-1 / 2} y\right) T\left(D^{-1 / 2} y^{\prime}, x^{\prime}\right)\right) \\
& =\left(D^{-1 / 2} y^{\prime}, D^{-1 / 2} y\right) \operatorname{Tr}\left(T\left(x, x^{\prime}\right)\right) \\
& =\left(x, x^{\prime}\right)\left(D^{-1 / 2} y^{\prime}, D^{-1 / 2} y\right)
\end{aligned}
$$

which is exactly as given in [2] and [4]. 


\section{REFERENCES}

1. A. Connes, Une classification des facteurs de type III, Ann. Ecole Norm. Sup. 4th series, 6 (1973), 133-252.

2. M. Duflo and C. C. Moore, On the regular representation of a non-unimodular locally compact group, J. Functional Analysis, 21 (1976), 209-243.

3. G. Pedersen and M. Takesaki, The Radon-Nikodym theorem for von Neumann algebras, Acta Math., 130 (1973), 53-87.

4. J. N. Phillips, A note on square integrable representations, J. Functional Analysis, 20 (1975), 83-92.

5. M. A. Rieffel, Square integrable representations of Hilbert algebras, J. Functional Analysis, 3 (1969), 265-300.

6. J. Rosenberg, Square integrable factor representations of locally compact groups, (to appear).

7. I. E. Segal, A noncommutative extension of abstract integration, Ann. of Math., 57 (1953), 401-457.

8. C. E. Sutherland, The direct integral theory of weights, and the Plancherel formula, Ph.D. dissertation, UCLA, 1973.

Received October 27, 1976. Supported in part by NSF grant MPS 74-19876.

UNIVERSITY OF CALIFORNIA

BERKELEY, CA 94720 



\section{PACIFIC JOURNAL OF MATHEMATICS \\ EDITORS}

RICHARD ARENS (Managing Editor)

University of California

Los Angeles, CA 90024

\section{R. A. Beaumont \\ University of Washington \\ Seattle, WA 98105}

C. C. MOORE

University of California

Berkeley, CA 94720

\section{J. DUGUNDJI}

Department of Mathematics

University of Southern California

Los Angeles, CA 90007

R. FINN AND J. MILGRAM

Stanford University

Stanford, CA 94305

\section{ASSOCIATE EDITORS}

\section{E. F. BECKENBACH}

B. H. NeUmanN

F. WOLF

K. YoSHIDA

\section{SUPPORTING INSTITUTIONS}

\author{
UNIVERSITY OF BRITISH COLUMBIA \\ CALIFORNIA INSTITUTE OF TECHNOLOGY \\ UNIVERSITY OF CALIFORNIA \\ MONTANA STATE UNIVERSITY \\ UNIVERSITY OF NEVADA \\ NEW MEXICO STATE UNIVERSITY \\ OREGON STATE UNIVERSITY \\ UNIVERSITY OF OREGON \\ OSAKA UNIVERSITY
}

\author{
UNIVERSITY OF SOUTHERN CALIFORNIA \\ STANFORD UNIVERSITY \\ UNIVERSITY OF HAWAII \\ UNIVERSITY OF TOKYO \\ UNIVERSITY OF UTAH \\ WASHINGTON STATE UNIVERSITY \\ UNIVERSITY OF WASHINGTON \\ AMERICAN MATHEMATICAL SOCIETY
}

The Supporting Institutions listed above contribute to the cost of publication of this Journal, but they are not owners or publishers and have no responsibility for its contents or policies.

Mathematical papers intended for publication in the Pacific Journal of Mathematics should be in typed form or offset-reproduced (not dittoed), double spaced with large margins. Underline Greek letters in red, German in green, and script in blue. The first paragraph or two must be capable of being used separately as a synopsis of the entire paper. Items of the bibliography should not be cited there unless absolutely necessary, in which case they must be identified by author and Journal, rather than by item number. Manuscripts, in duplicate, may be sent to any one of the four editors. Please classify according to the scheme of Math. Reviews, Index to Vol. 39. All other communications should be addressed to the managing editor, or Elaine Barth, University of California, Los Angeles, California, 90024.

100 reprints are provided free for each article, only if page charges have been substantially paid. Additional copies may be obtained at cost in multiples of 50 .

The Pacific Journal of Mathematics is issued monthly as of January 1966. Regular subscription rate: $\$ 72.00$ a year (6 Vols., 12 issues). Special rate: $\$ 36.00$ a year to individual members of supporting institutions.

Subscriptions, orders for back numbers, and changes of address should be sent to Pacific Journal of Mathematics, 103 Highland Boulevard, Berkeley, California, 94708.

PUBLISHED BY PACIFIC JOURNAL OF MATHEMATICS, A NON-PROFIT CORPORATION

Printed at Jerusalem Academic Press, POB 2390, Jerusalem, Israel.

Copyright (C) 1977 Pacific Journal of Mathematics All Rights Reserved 


\section{Pacific Journal of Mathematics \\ Vol. 70, No. $2 \quad$ October, 1977}

B. Arazi, A generalization of the Chinese remainder theorem ........... 289

Thomas E. Armstrong, Polyhedrality of infinite dimensional cubes .... . . . 297

Yoav Benyamini, Mary Ellen Rudin and Michael L. Wage, Continuous

images of weakly compact subsets of Banach spaces ............ 309

John Thomas Burns, Curvature functions on Lorentz 2-manifolds ......... 325

Dennis F. De Riggi and Nelson Groh Markley, Shear distality and equicontinuity .................................. 337

Claes Fernström, Rational approximation and the growth of analytic

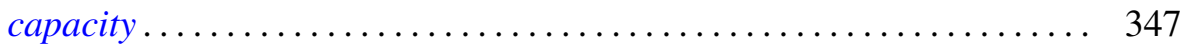

Pál Fischer, On some new generalizations of Shannon's inequality....... 351

Che-Kao Fong, Quasi-affine transforms of subnormal operators ......... 361

Stanley P. Gudder and W. Scruggs, Unbounded representations of

*-algebras........................................ 369

Chen F. King, A note on Drazin inverses .................... 383

Ronald Fred Levy, Countable spaces without points of first countability . . . 391

Eva Lowen-Colebunders, Completeness properties for convergence

spaces ......................................... 401

Calvin Cooper Moore, Square integrable primary representations ....... 413

Stanisław G. Mrówka and Jung-Hsien Tsai, On preservation of

E-compactness ................................ 429

Yoshiomi Nakagami, Essential spectrum $\Gamma(\beta)$ of a dual action on a von

Neumann algebra ................................ 437

L. Alayne Parson, Normal congruence subgroups of the Hecke groups

$G\left(2^{(1 / 2)}\right)$ and $G\left(3^{(1 / 2)}\right)$...

Louis Jackson Ratliff, Jr., On the prime divisors of zero in form rings . . . . 489

Caroline Series, Ergodic actions of product groups .................. 519

Robert O. Stanton, Infinite decomposition bases..................... 549

David A. Stegenga, Sums of invariant subspaces .................. 567 